\title{
Comparison of different extraction methods for the extraction of major bioactive flavonoid compounds from spearmint (Mentha spicata L.) leaves.
}

\begin{abstract}
Different bioactive flavonoid compounds including catechin, epicatechin, rutin, myricetin, luteolin, apigenin and naringenin were obtained from spearmint (Mentha spicata L.) leaves by using conventional soxhlet extraction (CSE) and supercritical carbon dioxide (SC-CO2) extraction at different extraction schemes and parameters. The effect of different parameters such as temperature (40, 50 and $60{ }^{\circ} \mathrm{C}$ ), pressure $(100,200$ and 300 bar) and dynamic extraction time $(30,60$ and $90 \mathrm{~min}$ ) on the supercritical carbon dioxide (SC-CO2) extraction of spearmint flavonoids was investigated using full factorial arrangement in a completely randomized design (CRD). The extracts of spearmint leaves obtained by CSE and optimal SC-CO2 extraction conditions were further analyzed by high performance liquid chromatography (HPLC) to identify and quantify major bioactive flavonoid compounds profile. Comparable results were obtained by optimum SC-CO2 extraction condition $\left(60{ }^{\circ} \mathrm{C}\right.$, 200 bar, $60 \mathrm{~min}$ ) and $70 \%$ ethanol soxhlet extraction. As revealed by the results, soxhlet extraction had a higher crude extract yield $(257.67 \mathrm{mg} / \mathrm{g})$ comparing to the SC-CO2 extraction $(60.57 \mathrm{mg} / \mathrm{g})$. Supercritical carbon dioxide extract (optimum condition) was found to have more main flavonoid compounds (seven bioactive flavonoids) with high concentration comparing to the $70 \%$ ethanol soxhlet extraction (five bioactive flavonoids). Therefore, SC-CO2 extraction is considered as an alternative process compared to the CSE for obtaining the bioactive flavonoid compounds with high concentration from spearmint leaves.
\end{abstract}

Keyword: Spearmint (Mentha spicata L.); Bioactive flavonoid; Supercritical carbon dioxide (SC-CO2) extraction; Conventional soxhlet extraction (CSE); High performance liquid chromatography (HPLC). 